\title{
On the use of sub-harmonic mixer in sliding-IF architecture for mm-Wave/THz transceivers
}

\author{
Sumit Pratap Singh, Mostafa Jafari Nokandi, Timo Rahkonen, Aarno Pärssinen \\ Faculty of Information Technology \\ and Electrical Engineering \\ University of Oulu \\ Oulu, Finland \\ first name.last name@oulu.fi
}

\begin{abstract}
This paper discusses methods to implement a sub$\mathrm{THz}$ transceiver for $300 \mathrm{GHz}$ band with minimal LO frequency. An existing sub-harmonic mixer, used previously in direct conversion transceiver, is applied in a sliding-IF mixer architecture. Sub-harmonic mixer with sliding IF architecture is envisioned to further reduce the need of higher LO frequency for upconversion. In this aspect, two architectures are studied by spectral analysis and further verified through circuit simulator. The first has a problem with overlapping sidebands, making it unsuitable for non-symmetrical double sideband modulations. On the other hand, the second architecture manages to up-convert signals up to $f_{o}$, using a single LO frequency of $f_{o} / 3$.

Index Terms-Mixer, Gilbert Cell, Sub-harmonic, sliding-IF, mm-Wave, THz, transceiver
\end{abstract}

\section{INTRODUCTION}

Frequency spectrum above $100 \mathrm{GHz}$ is most promising for beyond 5G application. The availability of wide-band and unused spectrum above $100 \mathrm{GHz}$ inspires to build ultra high-speed next generation wireless transceiver, imaging and sensing [1]-[4]. Transceivers well above $100 \mathrm{GHz}$ are capable of handling datarates of more than $100 \mathrm{~Gb} / \mathrm{s}$. IEEE standard 802.15.3d in the $300 \mathrm{GHz}$ band has been established [5]. Considering this, the use of WR-3 waveguide band $(220 \mathrm{GHz}$ $320 \mathrm{GHz}$ ) is the most suitable for ultra high speed transceivers.

For any transceiver, one of the most important aspects is its RF front-end architecture. The choice of a RF front-end architecture is not only limited by its RF performance but other aspects such as complexity, cost and power dissipation [6]. Modern fast IC technologies such as SiGe BiCMOS and IIIV HEMT are capable of supporting $\mathrm{mm}-\mathrm{Wave} / \mathrm{THz}$ circuits. However, power generation at $\mathrm{THz}$ frequency range is one of the most difficult aspects of transceiver design. Traditionally, there are only two ways to generate high frequency LO power: one is directly from the oscillators and other is by frequency multiplier chain. In [7], it is seen that the frequency multiplier chain is not only the most power hungry portion of any $\mathrm{THz}$ transceivers but it also occupies a large area. SlidingIF architecture is a heterodyne architecture, where the LO frequencies track each other, so that they can be generated from the same source. However, at $\mathrm{THz}$ frequencies, slidingIF also requires the use of multiplier chain. In this paper, we target in mixing the signal up to $300 \mathrm{GHz}$ by using as low

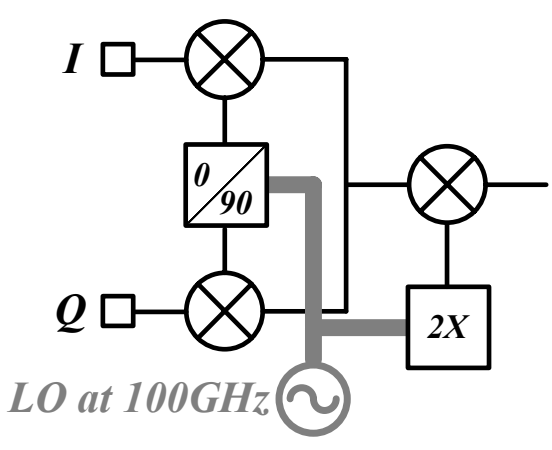

Fig. 1. Sliding-IF architecture.

LO frequency as possible. For this purpose we study two novel topologies, where a sub-harmonic mixer is used in a sliding-IF architecture. Two possible architectures are analysed, and one is discarded because harmonic mixing is causing aliasing of the sidebands. The other architecture, however, is capable of upconverting signal from baseband to $300 \mathrm{GHz}$ by using only a $100 \mathrm{GHz} \mathrm{LO}$.

\section{APPLICABILITY OF SLIDING IF ARCHITECTURE IN MM-WAVE AND THZ TRANSCEIVER}

Generation of multiple LO frequencies is costly, and using just one frequency source would be preferred. Sliding-IF architecture, as shown in Fig. 1, is one of the most suitable architectures for $\mathrm{mm}$-Wave/THz transceivers. It has been gaining popularity for two reasons: first, the LO frequency is still considerably lower than the RF frequency, and second the two LO frequencies are tracking each other, so one can easily be generated from the other one.

Fig. 1 shows the initial architecture for a $300 \mathrm{GHz}$ transmitter. The baseband is first modulated up to $100 \mathrm{GHz}$ with an IQ-modulator. Then it is further up-converted to $300 \mathrm{GHz}$ by effectively multiplying it by $200 \mathrm{GHz} \mathrm{LO}$ signal. However, the LO signal generation is very demanding at $\mathrm{mm}-\mathrm{Wave} / \mathrm{THz}$. Due to low gain of both multipliers and gain stages, the LO signal chain contains multiple buffer amplifiers, or sometimes 


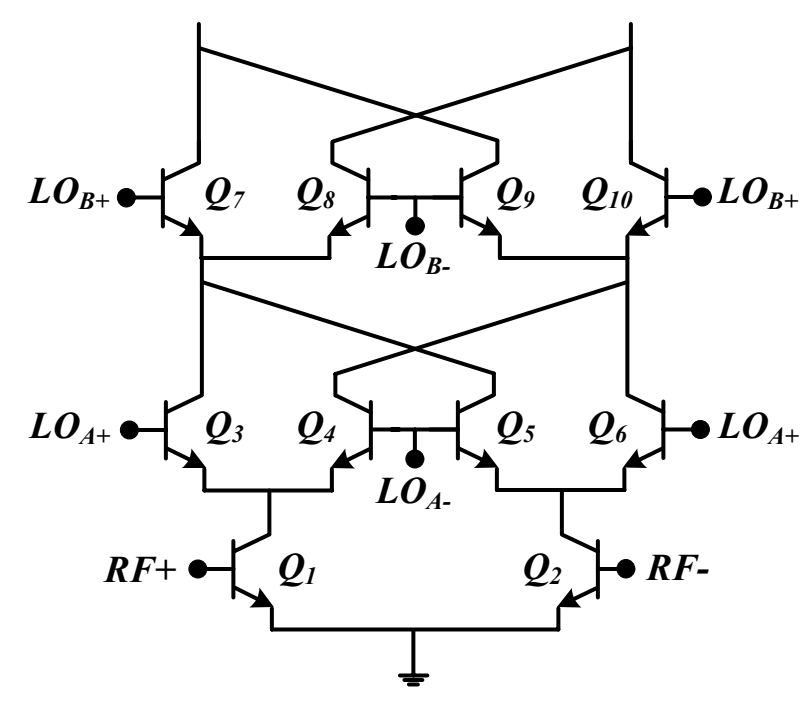

(a)

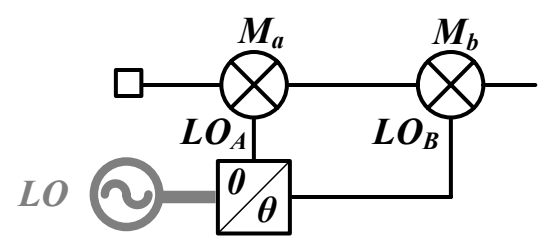

(b)

Fig. 2. 3 level Gilbert Cell (a) Schematic (b) Block Diagram

power amplifier, to boost the signal power level so that they can drive hard the mixer's LO port. To overcome this problem, possible use of existing sub-harmonic mixer are analysed in the next section.

\section{3-LEVEl Gilbert CELl BASED Sub-HARMONIC MIXER}

Direct conversion receivers suffer from many problems such as DC offset, even-order distortion and flicker noise [6]. However, two level mixing in sub-harmonic mixer resolves some of these problems in direct conversion receivers. Recently sub-harmonic mixer in direct conversion architecture is implemented in $\mathrm{THz}$ frequency ranges [8]. Here, a popular '3-level Gilbert cell' is used as a sub-harmonic mixer [9]. The schematic of the three level Gilbert cell, as shown in Fig. 2(a), performs two level mixing, using two stacked switching quads (Q3-Q6 and Q7-Q10), both having the same LO frequency.

The effect of phase difference between the two LO inputs seems to be an important factor which largely affects the conversion gain in both up- and down-converter mixers. In [10], phase difference error and duty cycle error in two LO inputs are analysed to observe the LO leakage to the RF input port. It is concluded that the LO rejection is relatively insensitive to the phase difference error, however, it is primarily a function of the duty cycle error. On the other hand, it is also reported that the in-phase LO signals provide zero conversion gain while quadrature LO signals provide a maximum conversion gain, assuming that the Gilbert cell based sub-harmonic mixer is driven with ideal square wave LO signal [8]. At mm-Wave/THz frequencies the harmonic mixing gains are smaller, but there still is noticeable gain difference. Considering this, this topology with sliding-IF architecture is thoroughly analysed in the next section.

\section{VERIFYING THE SLIDING IF ARCHITECTURES}

As shown in the Fig. 3, there are two possible ways to implement the sub-harmonic mixer topology with sliding-IF architecture in transmitter. One is putting the subharmonic mixer cell after I and Q signal up-conversion, and the other is using it in the I and Q mixing itself. In both ways, using only $100 \mathrm{GHz}$ of LO signal, a baseband signal with centre frequncy at $0 \mathrm{~Hz}$ can be converted to $300 \mathrm{GHz}$ signal.

During spectrum analysis of the architectures, it is observed that the topology in Fig. 3(a) suffers from spectrum overlap at the desired frequency which distorts the signal to be transmitted. This is depicted in Fig. 4(a), where the mixing process is explained. Here, $L_{1}$ and $L_{3}$ are the fundamental and 3rd harmonic mixing gains of the mixer at 100 and 300 $\mathrm{GHz}$, respectively. Signal S is the output spectrum of the BBto-100 GHz IQ modulator shown in Fig. 3(a). As a result of first mixing, at point node $\mathrm{B}$, it is observed that the $L_{3}$ at 300 $\mathrm{GHz}$ causes the signal and its spectrally reversed image at the negative frequency to overlap at $200 \mathrm{GHz}$. From Taylor series of a square wave mixing function, $L_{3}$ is one third and out of phase with $L_{1}$. So the product $L_{3} S^{*}$ can severely distort the the desired signal $L_{1} \mathrm{~S}$ at $200 \mathrm{GHz}$, as their difference is only about $10 \mathrm{~dB}$.

The signal at $200 \mathrm{GHz}$ gets distorted further when it is again multiplied by LO signal in the upper quad of the 3 level Gilbert mixer. Now, the desired signal at $300 \mathrm{GHz}$ is further distorted by the 3rd harmonic of LO which mixes both the $400 \mathrm{GHz}$ and the DC band results to $300 \mathrm{GHz}$. The resulting up-converted signal magnitude can be expressed as shown in (1)

$$
\begin{aligned}
S L O_{1} L O_{2}= & L_{1}\left(L_{3} S^{*}+L_{1} S\right)+L_{1}^{*} L_{3} S \\
& +L_{3}\left(L_{1} S^{*}+L_{1}^{*} S\right)
\end{aligned}
$$

Consequently, this phenomenon causes degradation in SNR of the transmitting signal. Hence, it is suited only for transmitting modulations with symmetrical sidebands such as AM and ASK. Alternatively, the mixers must be used in linear multiplying mode, so that the third harmonic gain $L_{3}$ do not mix the troublesome sidebands. However, in practice it is difficult to guarantee such linearity.

This effect was also verified by simulation using VBIC model of SiGe BiCMOS process technology with $f_{\max }$ of $450 \mathrm{GHz}$. In simulation verification, it should be noted that $\mathrm{CW}$ harmonic balance either at the center of the band or any modulation with symmetrical spectrum (e.g. AM modulation) does not reveal the overlapping of the spectral images, as they will be seen as a change in gain only. On the other 


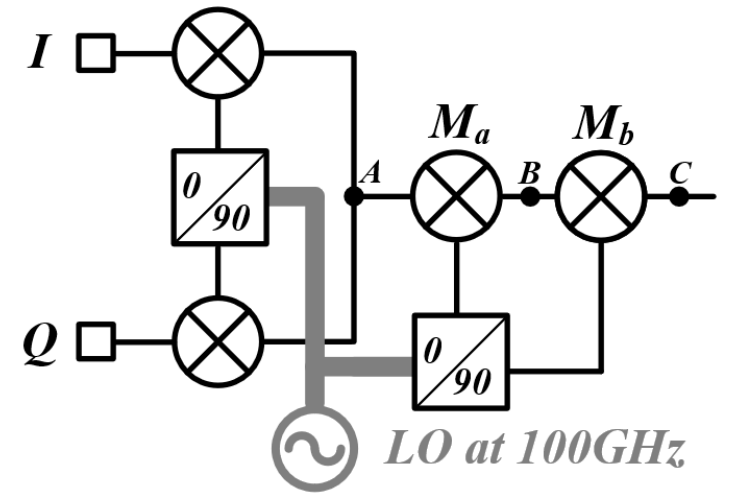

(a)

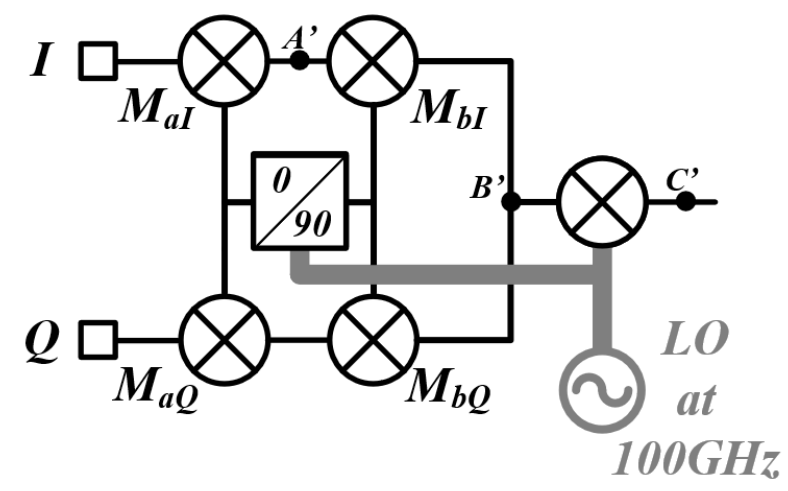

(b)

Fig. 3. Block diagram of Sliding-IF architecture with a) post sub-harmonic mixing b) pre sub-harmonic mixing
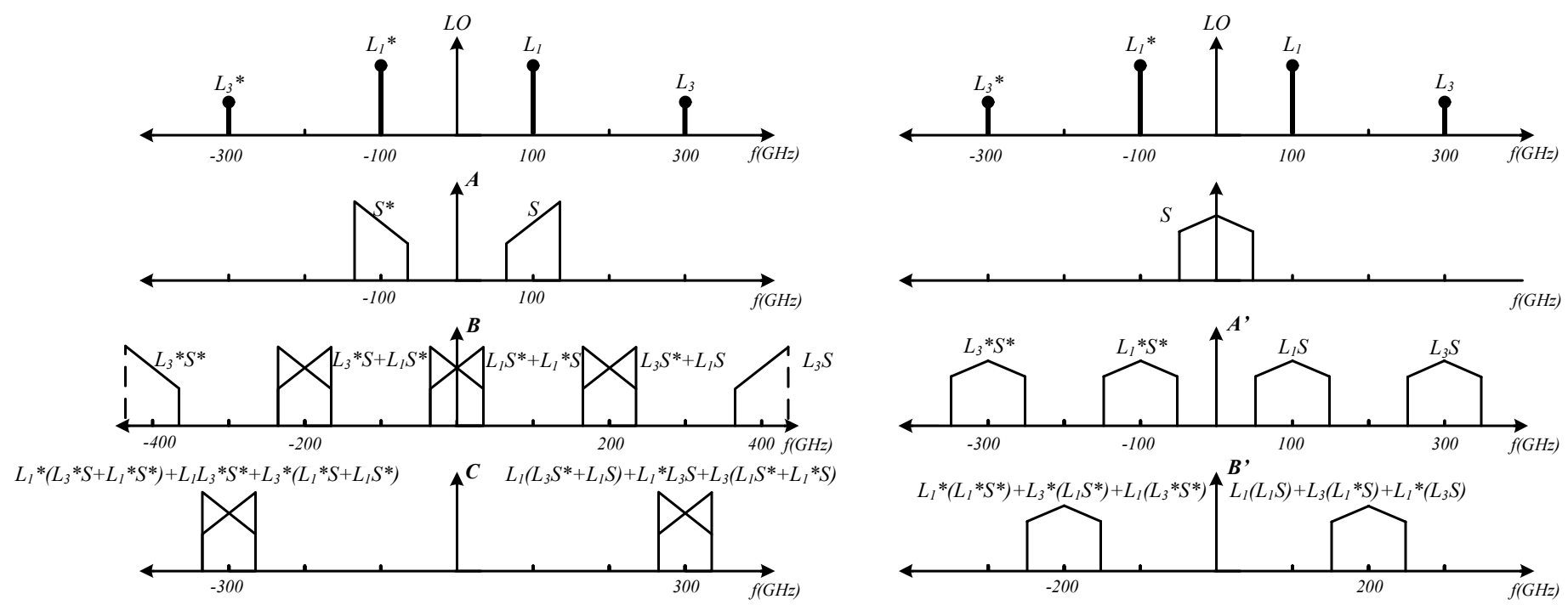

(a)

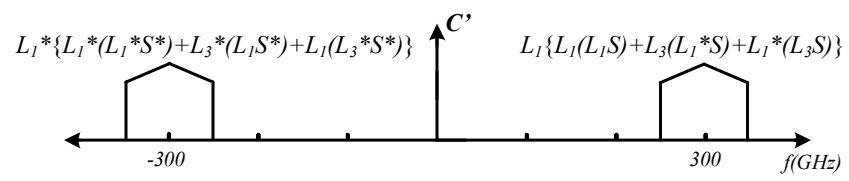

(b)

Fig. 4. Spectral analysis of Sliding-IF architecture with a) post sub-harmonic mixing b) pre sub-harmonic mixing

hand, asymmetrical modulation scheme clearly highlights this phenomenon.

In IQ modulators, the asymmetric modulation is most easily arranged by using sine and cosine as baseband I and Q signals. During up-conversion, they create just one side band. In sliding IF architecture, with post sub-harmonic mixing, simulation it is observed that single tone $100 \mathrm{GHz}$ signal can easily be upconverted to $300 \mathrm{GHz}$ single tone. However, to make the IF spectrum symmetrical, a single tone at one side of the center frequency, say at $101 \mathrm{GHz}$ is considered as IF input to sub-harmonic mixer. In this case, it is observed that the upconverted output signal contains two single tones at $299 \mathrm{GHz}$ and $301 \mathrm{GHz}$ as shown in Fig. 5. As image band folds on top of the signal, undesired tone of $299 \mathrm{GHz}$ also appears in the output. Simulation result also depicts that the sideband rejection is only 6-10 dB, which is not sufficient for any asymmetric modulation. Hence, the proposed sliding-IF architecture with post sub-harmonic mixing does not fulfil the requisite criteria.

In the second architecture, sub-harmonic mixing of basband signal, using three level Gilbert cell, is performed in I and Q branch itself as shown in the Fig. 3(b). Here, LO signal of $100 \mathrm{GHz}$ up-converts baseband I and Q signals to an output signal at $200 \mathrm{GHz}$ with sufficient sideband rejection. A further mixing with $100 \mathrm{GHz}$ LO moves the signal up to $300 \mathrm{GHz}$. As depicted in Fig. 4(b), harmonic mixing does not affect this architecture. Only the fifth harmonic $\left(L_{5}\right)$ would mix the sidebands on top of each other, however, that is already at low 


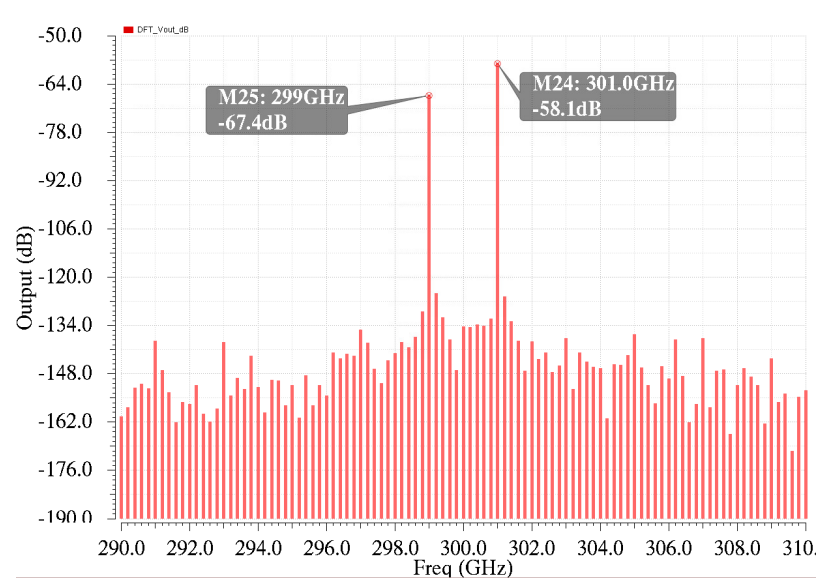

Fig. 5. Output spectrum when $101 \mathrm{GHz}$ is upconverted with two-level subharmonic mixer to $301 \mathrm{GHz}$

level. Hence, this architecture which uses five mixing stages, it makes possible to mix BB signals up to $300 \mathrm{GHz}$ by using only $100 \mathrm{GHz} \mathrm{LO}$ in all the mixer cells.

\section{DISCUSSION}

In this paper, we took a fresh look on a sub-harmonic mixer architecture that has been considered for direct conversion applications in the past. In [10], LO leakage (due to duty cycle asymmetry) is analysed. In [8], it is observed that the harmonics of the mixing function can be used for gain boosting at low frequencies (in which case 90 degree phase shift between the LOs is needed), but approaching maximum frequency of oscillation of transistor, the effect of harmonics fades away.

Here, three-level Gilbert cell based sub-harmonic mixer is proposed in two possible configurations with sliding IF architecture . In both of them, the LO would be just $1 / 3$ of the $\mathrm{RF}$ frequency. It seems that the architecture where a normal IQ modulator is followed by the mixer, where all are sharing the same LO frequency, is not suited for this purpose due to poor sideband rejection caused by the frequency-reversed image of the spectrum that falls on top of the desired signal. This conclusion is verified by both spectral analysis and circuit simulations. In either analysis, it is essential that the IF spectrum used in the analysis is non-symmetrical so that we can see the side-band aliasing in the output.

On the other hand, the second sliding-IF architecture with pre sub-harmonic mixing in the IQ branch does not encounter the above problem and this architecture turns out be a better option for mm-Wave/THz transceiver with single LO frequency which is $1 / 3$ of the RF frequency.

\section{REFERENCES}

[1] T. S. Rappaport et al., "Wireless Communications and Applications above $100 \mathrm{GHz}$ : Opportunities and Challenges for 6G and Beyond," IEEE Access, vol. 7, July 2019, pp. 78729-57

[2] U. R. Pfeiffer, R. Jain, J. Grzyb, S. Malz, P. Hillger and P. RodríguezVízquez, "Current Status of Terahertz Integrated Circuits From Components to Systems," 2018 IEEE BiCMOS and Compound Semiconductor Integrated Circuits and Technology Symposium (BCICTS), San Diego, CA, USA, 2018, pp. 1-7.
[3] Koenig, S. et al. Wireless sub-THz communication system with high data rate. Nat. Photon 7, 977-981 (2013).

[4] Fujishima, Minoru, "Key technologies for THz wireless link by silicon CMOS integrated circuits," In Photonics, vol. 5, no. 4, p. 50. Multidisciplinary Digital Publishing Institute, 2018.

[5] IEEE Standard for High Data Rate Wireless Multi-Media Networks, Amendment 2: $100 \mathrm{~Gb} / \mathrm{s}$ Wireless Switched Pointto-Point Physical Layer, IEEE Computer Society sponsored by the LAN/MAN standards committee. Available online: https://standards.ieee.org/standard/802.15.3d 2017.html (accessed on 21 November 2018).

[6] B. Razavi, RF Microelectronics, NJ, Upper Saddle River:Prentice-Hall, 2015.

[7] E. jefors, B. Heinemann, U. R. Pfeiffer, "Active 220- and 325-GHz frequency multiplier chains in a SiGe HBT technology", IEEE Trans. Microw. Theory Tech., vol. 59, no. 5, pp. 1311-1318, May 2011.

[8] Y. Zhao, E. Ojefors, K. Aufinger, T. Meister, U. Pfeiffer, "A 160$\mathrm{GHz}$ subharmonic transmitter and receiver chipset in an SiGe HBT technology", IEEE Trans. Microw. Theory Techn., vol. 60, no. 10, pp. 3286-3299, Oct. 2012.

[9] Choma J Jr. A three-level broad-banded monolithic analog multiplier. IEEE Journal of Solid-State Circuits 1981; SC-16(4):392-399.

[10] L. Sheng, J. C. Jensen, L. E. Larson, "A wide-bandwidth Si/SiGe HBT direct conversion sub-harmonic/downconverter", IEEE J. SolidState Circuits, vol. 35, pp. 1329-1337, Sept. 2000. 